This means that $x$ is expressible as

$$
\nu \varepsilon^{k}=x=\lambda_{1}^{k}+\ldots+\lambda_{s}^{k}+\tau_{1}^{k}+\ldots+\tau_{2 s_{1}}^{k}
$$

subject to the conditions

$$
\lambda_{j} \in P(T), \quad \tau_{j} \in P\left(T_{1}\right),
$$

whence follows the desired result.

\title{
Zur Theorie der symplektischen Gruppen
}

ron

\section{References}

Urremor Cimastran (Göttingen)

[I] $\mathrm{X} . \mathrm{Eda}$. On the Waring problem in an algebraic number field, in Seminar on Modern Methods in Number Theory, Tokyo 1971.

[2] H. H asse, Vörlesungen ïher Klassenkörpertheorie, Würzburg 1967.

[3] L. K. Hua, Exponential sums over algebraic fields, Canadian J. Math. 3 (1951), pp. $44-51$.

[4] - On Waring's problem, Quart. Journ. of Math. 9 (1938), pp. 199-201.

[5] 0. Körner, Utber das Waringsehe Problem in algebraischen Zahllkörpern, Math. Ann. 144 (1961), pp. 224-238.

[6] E. Landau, Uber die neue Winogradoffsche Behandlung des Waringsohen Problems, Math. Zeitschr. 31 (1930), pp. 319-338.

[7] T. Mitsui, On the Goldbach problem in algebraio number field $I$, Journ. Math. Soc. Japan 12 (1960), pp. 325-372.

[8] C. L. Siegel, Generalization of Waring's problem to algebraic number fields, Amer. Journ. Math. 66 (1944), pp. 122-136.

[9] - Sums of m.th powers of algebraio integers, Ann. of Math. 46 (1945), pp. 313339.

[10] R. M. Stemmler, The easier Waring problem in algebraic number fields, Acta Arith. 6 (1961), pp. 447-468.

[11] T. Takagi, Algebraic number theory (in Japanese), Tokyo 1948.

[12] T. Tatuzawa, On the Waring proble in an algebraio number field, Journ. Math. Soc. Japan 10 (1958), pp. 322-31.

[13] - On the Waring problem in an algebraic number field, in Seminar on Modern Methods in Number Theory, Tokyo 1971.

[14] И. М. Витоградов, Ивбранные труды,. Москва 1952.

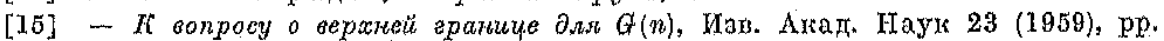
637-642.

INSTITUTE OF MATHMMATIC, COKLTGE OF GENMRAL WOUOATION UNIVERSTTY OF TOTYY

Garl Ludwig Siegel zum 75. Geburtstag und zum 50-jährigen Professorenjubiläum gewidmet

Will man den Rang der Schar der Modulformen mit Hilfe der Selbergschen Spurformel berechnen, so stößt man auf ein Problem, welches man grob so beschreiben kann: Es sei $M$ eine Modulmatrix $n$-ten Grades. Wann gibt es eine Modulmatrix $n$-ten Grades $R$, so daß die Matrix $R^{-1} M R$ eine Randkomponente der verallgemeinerten oberen Halbebene im Unendlichen festläßßt?

Zur Liösung dieses Problems führen wir zunächst einige Bezeichungen ein.

1. Man bilde dio $(2 n) \times(2 n)$ Matrix

$$
I(n)=\left(\begin{array}{rr}
0 & E \\
-D & 0
\end{array}\right)
$$

mit $n \times n$ Null- bzw. Einheitsmatrix 0 und $E$. Für einen Körper $K$ bezeichnen wir mit $\Sigma(n, K)$ die symplektische Gruppe, bestehend aus allen $(2 n) \times(2 n)$ Matrizen $M$ mit Elementen in $K$, die der Bedingung

$$
M^{\prime} I(n) \not M=I(n)
$$

genügon. Fiorbei bedentot $M^{\prime}$ dio Transponierte von $M$. Zwei Matrizen $M, M^{*} \in \Sigma(n, K)$ heißen konjugiert über $K$, wenn es ein $R \in \Sigma(n, K)$ mit

$$
R^{-1} M R=M^{*}
$$

gibt. Es seien $M, R \in \Sigma(n, K)$. Wir sagen, $d a B R^{-i} M R$ aus $M$ hervorgeht, indem man $M$ mit $R$ "konjugiert". In der üblichen Weise bezeichne man mit $\boldsymbol{Q}, \boldsymbol{C}$ die Körper der rationalen, bzw. komplexen Zahlen. Für diese gesamte Arbeit gelte

$$
Q \subset K \subset C
$$


Die Siegelsche Modulgruppe n-ten Grades $T(n)$ ist diejenige Unter gruppe von $\Sigma(n, Q)$, die aus allen Matrizen mit ganzzahligen Elementen besteht. Jetizt sei $\Psi$ eine Untergruppe von $\Sigma(n, C)$ und $j$ eine natürliche Zahl mit $0 \leqslant j \leqslant n$. Dann bezeichne $\Psi_{j}$ die Menge aller Matrizen aus $\Psi$ von folgender Gestalt:

$$
M=\left(\begin{array}{cccc}
A_{j} & 0 & B_{j} & * \\
* & P_{j}^{\prime} & * & * \\
O_{j} & 0 & D_{j} & * \\
0 & 0 & 0 & P_{j}^{-1}
\end{array}\right)
$$

Hierbei sind $A_{j}, B_{j}, C_{j}, D_{j}$ Matrizen mit $(n-j)$ Zeilen und Spalten and $P_{j}$ eine $j \times j$ Matrix. Die Gruppen $\Gamma_{j}(n)(1 \leqslant j \leqslant n)$ sind genau diejenigen die Randkomponenten der verallgemeinerten oberen Halbebene bei Unendlich festlassen.

Es sei $M$ eine quadratische Matrix. Dann bezeichnen wir mit

$$
\chi(M, x)=\operatorname{Det}(x E-M)
$$

das charakteristische Polynom von $M$. Ist insbesondere $M \in \Sigma(n, C)$, so gilt

$$
\chi(M, x)=x^{2 n} \chi\left(M, \frac{1}{x}\right)
$$

Zum Beweis siehe man [2, (13)]. Ein Polynom, welches dem Transformationsgesetz (7) genügt, wollen wir in dieser Arbeit "involutorisch" nennen.

Meine Arbeit [2] ist die Grundlage der vorliegenden Ontersuchung. Daher werde ich mich weiterhin eng an die Bezeichnung und die Resultate von [2] halten. Die Arbeit [2] steht auch im Zusammenhang mit einer Untersuchung von G. E. Wall [6].

Nunmehr beweisen wir folgende Resultate:

SATZ 1. Es sei $K$ ein Körper, welcher der Bedingung (4) genügt und $T$. eine $n \times n$ Matrix mit Elementen aus $K$. Angenommen, es gibt eine nichtsinguläre $n \times n$ Matrix $V$ mit Elementen aus $K$, so daß

$$
V^{-1} T V=\left(\begin{array}{ll}
T_{1} & T_{12} \\
0 & L_{2}
\end{array}\right)
$$

mit einer $r \times r$ Matrix $T_{1}$ und einer $(n-r) \times(n-r)$ Matrix $T_{2}$ gilt. Dann zerfallt das charalkteristische Polynom von $T$ im Polynomring $K[$ [W] in der Gestalt

$$
\chi(T, x)=k_{1}(x) k_{2}(x)
$$

mit

$$
\chi\left(T_{t}, x\right)=k_{\imath}(x) \quad(\iota=1,2)
$$

Die Polynome $k_{1}(x), k_{2}(x)$ haben also den höchsten Koeffizienten 1 , und es gilt

$$
\operatorname{Grad} k_{1}(x)=r, \quad \operatorname{Grad} k_{2}(x)=n-r .
$$

Nun zerfalle umgekehrt das charakteristische Polynom $\chi(T, x)$ in $K[x]$ in der Gestalt (9), so daß (11) gilt. Dann gibt es eine nicht-singuläre $n \times n$ Matrix $V$ mit Elementen aus $K$, derart daß (8) und (10) erfüllt sind. Es seien insbesondere die Ilemente von $T$ rational. Genau dann sind die Bedingungen, (8), (1.0) durch ein unimodulares $V$ mit $\operatorname{Det} V=1$ lösbar, wenn sie mit einem rationalen $V$ lösbar sind.

Satz 2. Ds sei $M \in \Sigma(n, K)$. Angenommen, es gibt ein $R \in \Sigma(n, K)$, so $d a \beta$

$$
R^{-1} M R=\left(\begin{array}{cccc}
A_{j} & 0 & B_{j} & * \\
* & P_{j}^{\prime} & * & * \\
C_{j} & 0 & D_{j} & * \\
0 & 0 & 0 & P_{j}^{-1}
\end{array}\right) \in \Sigma_{j}(n, K)
$$

mit $(n-j) \times(n-j)$ Matrizen $A_{-}, B_{j}, C_{j}, D_{j}$ und einer $j \times j$ Matrix $P_{j}$ gilt. Dann zerfällt das charakteristische Polynom von $M$ im Polynomring $K[x]$ in der Gestalt

mit

und

$$
\chi(M, x)=g(x) h(x) h^{*}(x)
$$

$$
\chi\left(M_{j}, x\right)=g(x)
$$

hierbei wurde

$$
\chi\left(P_{j}^{\prime}, x\right)=h(x), \quad \chi\left(P_{j}^{-1}, x\right)=h^{*}(x)
$$

gesetat. Die Polynome $g(x), h(x), h^{*}(x)$ haben also die höchsten Koeffizienten 1 , und es gilt

$$
\operatorname{Grad} g(x)=2(n-j), \quad \operatorname{Grad} h(x)=\operatorname{Grad} h^{*}(x)=j,
$$

sowie

und

$$
g(x)=x^{2(n-3)} g\left(\frac{1}{x}\right)
$$

Insbesondere ist also $g(x)$ involutorisch. 
Nehmen wir weiter an, es sei $p(x)$ ein involutorisches Polynom mit (20)

$$
\text { Grad } p(x)=2 c \text {, }
$$

wie es in $[2,(73)]$, beschrieben wird. $p(x)$ gehe in $\chi(M, x)$ genau zur s-ten Potenz und in $h(x)$ genau zur t-ten Potenz auf. Dann geht $p(x)$ auch in $h^{*}(x)$ genau zur t-ten Potenz auf.

Vermöge der in [2] entwickelten Reduktionstheorie ist dann M Tonjugiert zu einer Matrix

$$
\{\tilde{M}, \tilde{\ddot{M}}\} \in \Sigma(c s, n-c s, K)
$$

wobei die Matrizen $\tilde{M}, \tilde{\tilde{M}}$ bis auf Konjugiertenbildung eindeutig bestimmt sind. Die Bezeichnung $\Sigma\left(n_{1}, \ldots, n_{r} ; K\right)$ wurde in [2, Seite 217], definiert. Ferner gilt

$$
\chi(\tilde{M}, x)=p^{s}(x)
$$

während $\chi(\tilde{\tilde{M}}, x)$ zu $p(x)$ teilerfremd ist. Indem man $P_{j}$ mit einer nichtsingulären $j \times j$ Matrix konjugiert, deren Dlemente in $K$ liegen, kann man es in die Gestalt

$$
\left(\begin{array}{ll}
\tilde{P} & 0 \\
0 & \tilde{\tilde{P}}
\end{array}\right)
$$

mit einer $(20 t) \times(2 c t)$ Matrix $\tilde{P}$ und einer $(j-2 c t) \times(j-2 c t)$ Matrix $\tilde{\tilde{P}}$ transformieren. Hierbei gilt

$$
\chi(\tilde{P}, x)=p^{t}(x),
$$

während $\chi(\tilde{\tilde{P}}, x)$ zu $p(x)$ teilerfremd ist.

Aus der Lösbarkeit von (12) durch ein $R \in \Sigma(n, K)$ ergibt sich dann. weiter: Es existiert eine (2cs) $\times(2 c t)$-Matrix $N$ mit Elementen aus $K$, so daß folgendes gilt:

$$
\begin{gathered}
\tilde{M} N=N \tilde{P}, \\
N^{\prime} I(c s) N=0, \\
\operatorname{Rang} N=2 o t
\end{gathered}
$$

Jetzt nehmen wir umgekehrt an, daß das charakteristische Potynom von $M$ in der Gestalt (1.3) mit (17), (18), (1.9) zerfält. Ferner sei $p(x)$ ein in $K[x]$ irreduaibles involutorisches Polynom der vorher genanniten An, welches in $\chi(M, x)$ genau von s-ter und in $h(x)$ genau von t-ter Potens aufgeht. Wir wählen $z u p(x)$ eine $(2 c t) \times(2 c t)$ Matrix $\tilde{P}$ mit Elementen aus $K$, so aaß (24) gilt. Weiter transformieren wir $M$ in die Gestalt (21). . Dieses machen wir für jedes in $\chi(M, x)$ aufgehende involutorische Polynam $p(x)$ des oben genannten Typs. Und für jedes solche Polynom $p(x)$ mit der dazugewähtten Matrix $\tilde{P}$ seien die Bedingungen (25), (26), (27) durch ein $N$ lösbar.
Dann gibt es ein $R \in \Sigma(n, K)$, so daß die Bedingungen (12), (14), (15) gelten. Ist weiter $p(x)$ eines der oben genannten involutorischen Polynome und $\tilde{P}$ die dazugewählte Matrix, so 7ann man die in (12) auftretende Matrix $P_{\text {, }}$ duroh Konjugieren mit einer nicht-singulären $j \times j$ Matrix, deren Elemente aus $K$ sind, in die Gestalt (23) transformieren. Hierbei ist $\chi(\tilde{P}, x) \approx u p(x)$ teilerfremd.

Ds sei insbesondere $M \in \Sigma(n, Q)$. Genau dann sind die Bedingungen (12), (14), (15) duroh oine siegelsche Modulmatrix $R \in \Gamma(n)$ lösbar, wenn (12), (14), (15) durch ein $R \in \Sigma(n, Q)$ lösbar. sind.

Wix könnon also folgendes sagen. Abgesehen von den involutorischen Polynomen $p(x)$ ist (12) genau dann lösbar, wenn das charakteristische Polynom $\chi(M, x)$ in der Gestalt (13) mit (17), (18), (19) zerfällt. Bei den involutorischen Polynomen $p(x)$ lrommen noch die Bedingungen (25) bis (27) hinzu. Tch werde am Schluß der Arbeit auf die Lösbarkeit dieser Bedingungen, eingehen.

2. Beweis von Satz 1. Aus der Lösbarkeit von (8) durch eine Matrix $V$ folgen sofort die Bedingungen (9), (10), (11). Nun mögen umgekehrt (9), (11) gelten. Wir zeigen die Lösbarkeit von (8), (10) durch ein passendes $V$.

Ohne Beschränkung der Allgemeinheit kann man

$$
T=\left(\begin{array}{cccc}
B_{1} & & \\
& \ddots & 0 \\
& 0 & \ddots & \\
& & & B_{l}
\end{array}\right)
$$

annehmen mit

$$
\chi\left(B_{\lambda}, x\right)=j_{\lambda}^{b \lambda}(x) \quad(\lambda=1, \ldots, l),
$$

wobei $j_{\lambda}(x)$ ein irreduzibles Polynom in $K[x]$ und die $b_{\lambda}$ natürliche Zahlen sind. Ferner gelte

$$
\text { (30) } \operatorname{Grad} j_{\lambda}(x)=h_{\lambda}
$$

und

$$
\operatorname{Rang}\left(a_{\lambda} D-B_{\lambda}\right)=b_{\lambda} h_{\lambda}-1 \quad(\lambda=1, \ldots, l)
$$

für jede Nullstelle $\alpha_{\lambda}$ von $j_{\lambda}(x)$.

Offenbar gilt

$$
j_{1}^{b}(x) \ldots j_{l}^{b}(x)=\chi(T, x)=\chi_{1}(x) k_{2}(x) .
$$

Wie man leicht sieht, gibt es nicht-negative ganze Zahlen $b_{11}, \ldots, b_{l 1}$ and $b_{12}, \ldots, b_{22}$ miti

$(32) \quad \hat{b}_{6}(x)=j_{1}^{b_{1}}\left\langle(x) \ldots j_{l}^{b} l(x) \quad(\iota=1,2)\right.$
und

$$
b_{\lambda}=b_{\lambda 1}+b_{\lambda 2} \quad(\lambda=1, \ldots, l) .
$$

5 - Acta Arithmetica XXIV.I 
Angenommen, Satz 1 ist für jedes $B_{\lambda}$ richtig. Dann gibt os nicht-singuläre Matrizen $V_{\lambda}$ mit Elementen aus $K$ und ron derselben Zeilen- und Spaltenzahl wie $B_{\lambda}$, so da $\beta$

$$
V_{\lambda}^{-1} B_{\lambda} \nabla_{\lambda}=\left(\begin{array}{ll}
B_{\lambda 1} & B_{\lambda 12} \\
0 & B_{\lambda 2}
\end{array}\right) \quad(\lambda=1, \ldots, l)
$$

und

(35)

$$
\chi\left(B_{\lambda,}, x\right)=j_{\lambda}^{\partial_{\lambda \iota}}(x) \quad(\lambda=1, \ldots, l ; \iota=1,2)
$$

gilt. Hieraus folgt (8) mit

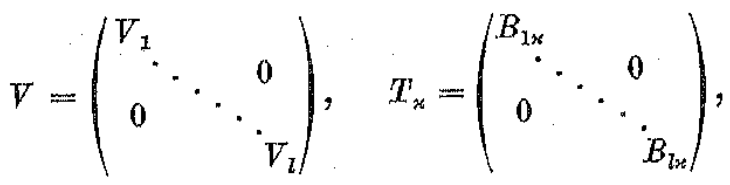

wobei $\varkappa$ die Indizes 1,2 und das bei $x_{12}$ auftretende Indexpaar 1,2 durchläuft. Es genügt also, die Lösbarkeit von (8) für den Fall $T=B$ mit

(36)

$$
\chi(B, x)=j^{b}(x)
$$

und

$$
\operatorname{Rang}(\alpha \not H-B)=b h-1
$$

zu zeigen. Hierbei ist $j(x)$ ein irreduzibles Polynom in $K[x]$ vom Gewicht $h$, ferner $b$ eine natürliche Zahl and a eine Nullstelle von $j(x)$. Wegen (9), (36) müssen auch $k_{1}(x)$ und $k_{2}(x)$ Potenzen von $j(x)$ sein, wobei aber auch die nullte Potenz zulässig ist.

Man sètze

$$
j(x)=x^{h}+a_{h-1} x^{h-1}+\ldots+a_{1} x+a_{0}
$$

und bilde die $h \times h$ Matrix

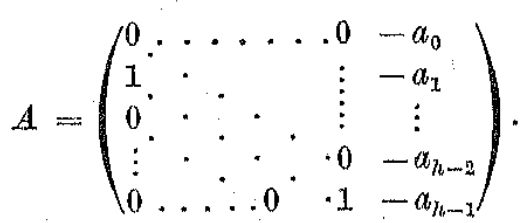

Die $(b h) \times(b h)$ Matrix $B$ ist dann za dex $(b h) \times(b h)$ Matrix

$$
\left(\begin{array}{cccc}
A . & B . & & \\
0 & \ddots & \cdot & 0 \\
0 & \cdot & \cdot & \cdot E \\
& & & \cdot A
\end{array}\right)
$$

Jetzt gelte (8) mit rationalen Matrizen $T, V$. Man setze

Hierbei ist $U$ unimodular, $\operatorname{Det} U=1$ und

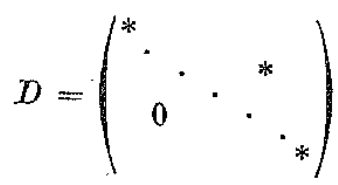

eine "Dreiecksmatrix". Ws folgt

$$
U^{-1} T U=D\left(\begin{array}{ll}
T_{1} & T_{12} \\
0 & T_{2}
\end{array}\right) D^{-1}
$$

Die rechte Seite von (4.2) besitzt wieder die Gestalt

Satz 1 ist bewiesen.

$$
\left(\begin{array}{ll}
* & * \\
0 & *
\end{array}\right)
$$

3. Beweis von Satz 2. Zuerst nehmen wir an, daß (12) gilt. Dann folgen sofort die Gleichungen (13) bis (19). Ferner wissen wir, daß $p(x)$ in $h(x)$ genau zur $t$-ten Potenz aufgeht. Da $p(x)$ involutorisch ist, folgt aus (19), daß $p(x)$ auch in $h^{*}(x)$ genau zur t-ten Potenz aufgeht. Nun transformiere man $M$ und $P_{j}$ in die Gestalt (21) bzw. (23). Ferner bringe man auch die durch (12), (16) definierte Matrix $M_{j}$ in die Gestalt

$$
M_{j}=\{\tilde{K}, \tilde{\tilde{K}}\} \epsilon \Sigma(c(s-2 t), n-j-c(s-2 t), \pi)
$$

und $\chi(\tilde{\tilde{K}}, x)$ teilexfremd zu $p(x)$. Durch rechnen sieht man, daß es eine Matrix $L \in \Sigma_{j}(n, K)$ mit

$$
I^{-1} R^{-1} M R L=\left(\begin{array}{cccccccc}
\tilde{A} & 0 & 0 & 0 & \tilde{B} & 0 & * & 0 \\
0 & \tilde{\tilde{A}} & 0 & 0 & 0 & \tilde{\tilde{B}} & 0 & * \\
* & 0 & \tilde{P}^{\prime} & 0 & * & 0 & * & 0 \\
0 & * & 0 & \tilde{P^{\prime}} & 0 & * & 0 & * \\
\tilde{O} & 0 & 0 & 0 & \tilde{D} & 0 & * & 0 \\
0 & \tilde{\tilde{O}} & 0 & 0 & 0 & \tilde{\tilde{D}} & 0 & * \\
0 & 0 & 0 & 0 & 0 & 0 & \tilde{P}^{-1} & 0 \\
0 & 0 & 0 & 0 & 0 & 0 & 0 & \tilde{P}^{-1}
\end{array}\right)
$$

Ironjugiert über $K$. Die Matrix (4.0) besitzt abor die auf der rechten Seite von (8) angegebene Gestalt. Damit ist Satz 1. bis auf die Schlußbemerkung über unimodulare Matrizen bewiesen. 
sowie

$$
\tilde{K}=\left(\begin{array}{cc}
\tilde{A} & \tilde{B} \\
\tilde{O} & \tilde{D}
\end{array}\right), \quad \tilde{\tilde{K}}=\left(\begin{array}{cc}
\tilde{\tilde{A}} & \tilde{\tilde{B}} \\
\tilde{\tilde{C}} & \tilde{\tilde{D}}
\end{array}\right)
$$

gibt. Die Sterne stehen für Elemente, die wir nicht näher zu kennen brauchen.

Man konjugiere die Matrix (45) mit

$$
\left(\begin{array}{ll}
U^{\prime} & 0 \\
0 & U^{-1}
\end{array}\right), \quad U=\left(\begin{array}{cccc}
E & 0 & 0 & 0 \\
0 & 0 & E & 0 \\
0 & E & 0 & 0 \\
0 & 0 & 0 & E
\end{array}\right)
$$

erreichen mit;

$$
J^{-1} \tilde{P}^{\prime} J=\tilde{P}
$$

gilt. Also kann man

$$
\tilde{M} \tilde{R}_{1}=\tilde{R}_{1}\left(\begin{array}{llll}
\tilde{p} & * & * & * \\
0 & * & * & * \\
0 & * & * & * \\
0 & * & * & *
\end{array}\right)
$$

$$
\tilde{R}_{1} \in \Sigma(c s, K)
$$

Es sei $N$ die (2cs) $\times(2 c t)$ Matrix, bestehend aus den ersten (2ct) Spaiten von $\tilde{R}_{1}$. Aus (53) folgt dann sofort (25). Nun geht $p^{t}(x)$ sowohl in $h(x)$ als auch in $h^{*}(x)$ auf. Wegen (13) also $p^{2 t}(x) \mid \chi(M, x)$, d.h., $2 t \leqslant s$. Hieraus und weil $\tilde{R}_{1}$ symplektisch ist, ergeben sich (26) und (27). Damit ist gezeigt, da $B$ aus der. Lösbarkeit von (1.2) alle in Satz 2 gennanten Bedingungen folgen.

4. Jetzt nehmen wir umgekehrt an, daß das charakteristische Polynom $\chi(M, x)$ in der Gestalt (13) zerfällt; dabei mögen (17), (18), (19) gelten. Ist ferner $p(x)$ ein involutorisches Polynom and $\tilde{P}$ eine zugehörige Matrix mit den in Satz 2 genannten. Eigenschaften, so seien (25), (26), (27) lösbar. Nun zeigen wir, daß es ein $R \in \Sigma(n, K)$ gibt, so daß die Bedingungen (12), (14), (1.5) gelten. Die in (12) auftretende Matrix $P_{j}$ kann man dabei in die Gestalt (23) bringen.

Zum Beweise gehen wir aus von der in [2] entwickelten Reduktionstheorie für symplektische Matrizen und bringen $M$ in die Gestalt

$$
M=\left\{M^{(1)}, \ldots, M^{(r)}\right\} \in \Sigma\left(n_{1}, \ldots, n_{r} ; K\right),
$$

wobei die charalkteristischen Polynome $\chi\left(M^{(1)}, x\right), \ldots, \chi\left(M^{(r)}, x\right)$ paarweise teilerfremd sind. Wir bilden die größten gemeinsamen Teiler

$$
\left\{\begin{array}{l}
g_{0}(x)=\left\langle g(x), \chi\left(M^{(0)}, x\right)\right\rangle, \\
h_{\ell}(x)=\left\langle h(x), \chi\left(M^{(e)}, x\right)\right\rangle, \\
h_{0}^{*}(x)=\left\langle h^{*}(x), \chi\left(M^{(e)}, x\right)\right\rangle
\end{array}\right.
$$

$(\varrho=1, \ldots, r)$ und setzen

$$
\text { (56) } \quad \operatorname{Grad} h_{Q}(x)=j_{\varrho} \quad(\varrho=1, \ldots, r) \text {. }
$$

Aus der Zerlegung $[2,(14)]$, folgt, daß jedes $g_{e}(x)$ involutorisch ist. Ferner gilt

$$
\operatorname{Grad} h_{e}^{*}(x)=j_{e} \quad(\varrho=1, \ldots, r)
$$


und

$$
h_{\varrho}^{*}(x)=\delta_{\varrho} x^{j_{e}} h_{\varrho}\left(\frac{1}{x}\right) \quad\left(0 \neq \delta_{Q} \in K ; \varrho=1, \ldots, r\right) .
$$

Ist $p(x)$ ein involutorisches Polynom der in Satz 2 genannten Art und sind $s$ und $t$ ebenfalls wie in Satz 2 definiert, so gibt es einen eindeutig bestimmten Index $\varrho=\varrho_{0}$, derart, daß $p(x)$ in $\chi\left(M^{\left(0_{0}\right)}, x\right)$ genau zur Potenz $s$ und in $h_{e_{0}}(x)$ und $h_{\varrho_{0}}^{*}(x)$ genau zur Potenz $t$ aufgeht, während $p(x)$ zu allen $\chi\left(M^{(e)}, x\right)$ mit $\varrho \neq \varrho_{0}$ teilerfremd ist. Schlieblich gilt

$$
\left\{\begin{aligned}
g(x) & =g_{1}(x) \ldots g_{r}(x), \\
h(x) & =h_{1}(x) \ldots h_{r}(x), \\
h^{*}(x) & =h_{1}^{*}(x) \ldots h_{r}^{*}(x),
\end{aligned}\right.
$$

$$
\chi\left(M^{(\varrho)}, x\right)=g_{\ell}(x) h_{\ell}(x) h_{e}^{*}(x) \quad\left(\varrho=1, \ldots, x^{*}\right) .
$$

Nehmen wir nun an, daß die Aussage von Satz 2 für jedes Kästchen $M^{(\varphi)}$ mit den zugehörigen Polynomen $g_{\varrho}(x), h_{\varrho}(x), h_{\varrho}^{*}(x)(\varrho=1, \ldots, r)$ richtig ist. Wir zeigen, daß dann Satz 2 auch für die Matrix $M$ gilt. Es sei nämlich

$$
R^{(\varrho)-1} M^{(\varrho)} R^{(Q)}=\left(\begin{array}{cccc}
A_{j_{Q}}^{(\varrho)} & 0 & B_{j_{Q}}^{(\varrho)} & * \\
* & P_{j_{Q}}^{(Q)} & * & * \\
\sigma_{j_{Q}}^{(\varrho)} & 0 & D_{j_{Q}}^{(\varrho)} & * \\
0 & 0 & 0 & P_{j_{Q}}^{(\varrho)-1}
\end{array}\right) \quad(\varrho=1, \ldots, r),
$$

$$
R^{(\varrho)} \in \Sigma\left(n_{a}, K\right) \quad(\varrho=1, \ldots, r)
$$

$$
\begin{aligned}
& M_{j_{e}}^{(\varrho)}=\left(\begin{array}{ll}
A_{j_{e}}^{(\varrho)} & B_{j_{e}}^{(\varrho)} \\
C_{j_{Q}}^{(\varrho)} & D_{j_{Q}}^{(\rho)}
\end{array}\right) \in \Sigma\left(n_{\varrho}-j_{\varrho}, K\right) \quad(\varrho=1, \ldots, r), \\
& \chi\left(M_{j_{e}}^{(\varrho)}, x\right)=g_{Q}(x) \quad(\varrho=1, \ldots, r), \\
& \text { (65) } \quad \chi\left(P_{\}_{Q}}^{()^{\prime}}, x\right)=h_{\ell}(x), \quad \chi\left(P_{j_{Q}}^{(\varrho)-1}, x\right)=h_{Q}^{*}(x) \quad(\varrho=1, \ldots, r) .
\end{aligned}
$$

Nun konjugiere man die Matrix

$$
\begin{aligned}
\left(R^{(1)-1} M^{(1)} R^{(1)}, \ldots, R^{(r)-1} M^{(r)} R^{(r)}\right) & \\
\quad & =\left(R^{(1)}, \ldots, R^{(r)}\right)^{-1}\left(M^{(1)}, \ldots, M^{(r)}\right)\left(R^{(1)}, \ldots, R^{(r)}\right)
\end{aligned}
$$

mit

$$
\left(\begin{array}{ll}
U^{\prime} & 0 \\
0 & U^{-1}
\end{array}\right)
$$

wobei die Elementie ron $U$ nur 0 oder 1 sind. Bei passender Wahl von $U$ erhält man (12) mit

$$
M_{j}=\left\{M_{j_{1}}^{(1)}, \ldots, M_{\}_{r}}^{(r)}\right\}
$$

und

$$
P_{j}=\left(\begin{array}{ccc}
P_{j_{1}}^{(1)} & & \ddots \\
0 & \ddots & 0 \\
0 & & \dot{P}_{j_{r}}^{(r)}
\end{array}\right) .
$$

Weiter sei $p(x)$ ein involutorisches Polynom der in Satz 2 genannten Art und $\tilde{P}$ die zugehörige Matrix. $p^{s}(x)$ teile $\chi\left(M^{\left(e_{0}\right)}, x\right)$, und es sei $p(x)$ zu $\chi\left(M^{(\varrho)}, x\right)\left(\varrho \neq \varrho_{0}\right)$ teilerfremd. Dann kann man $P_{j_{0_{0}}}^{\left(\varrho_{0}\right)}$ und infolgedessen auch $P_{j}$ in die Gestalt (23) transformieren. Damit sind alle Forderungen. von Satz 2 erfüllt.

Wegen $[2,(14)]$, genügt es also, Satz 2 für folgende Fälle zu beweisen:

$$
\chi(M, x)=q^{x}(x)
$$

wobei $q^{x}(x)$ wie in $[2, \S 3]$, definiert ist;

und

$$
\chi(M, x)=(x \pm 1)^{2 m}
$$

wobei $p^{s}(x)$ wie in $[2, \S 4]$, definiert ist.

5. Wir betrachten zuerst den Fall (68). Nach [2, (50)], hat man (71)

$$
q(x)=v(x) v^{*}(x)
$$

hierbei sind $v(x), v^{*}(x)$ irreduzible Polynome aus $K[x]$ mit dem höchsten Koeffizienten 1 und vom Grade $d$, für welche

$$
v^{*}(x)=\omega x^{d} v\left(\frac{1}{x}\right) \quad(0 \neq \omega \in K)
$$

gilt. Aus (13), (68), (71) folgt $h(x)=v^{\alpha}(x) v^{* \beta}(x)$ mit nicht-negativen ganzen Zahlen $\alpha$ und $\beta$. Wegen (19), (72) also $h^{*}(x)=v^{\beta}(x) v^{* a}(x)$. Offenbar genügt es nun, die Lösbarkeit von (12) durch ein passendes $R \in \Sigma(d \tau, K)$. für die Fälle $h(x)=v(x), h^{*}(x)=v^{*}(x)$ bzw. $h(x)=v^{*}(x), h^{*}(x)=v(x)$ zu boweisen, weil man daraus den allgemeinen Fall sofort durch Induktion ableiten kann. Bei $h(x)=v^{*}(x), h^{*}(x)=v(x)$ vertauschen wir noch die Bezejchnungen $v(\infty)$ and $v^{*}(\infty)$. Also genügt es, den Fall

$$
h(x)=v(x) \quad \text { und } \quad h^{*}(x)=v^{*}(x)
$$

zu betrachten.

Auf Grund von [2, Lemmai 3], gibt es ein $R_{1} \in \Sigma(d \tau, K)$ mit

$$
R_{1}^{-1} M R_{1}=\left(\begin{array}{ll}
T^{\prime} & 0 \\
0 & T^{-1}
\end{array}\right)
$$


und

$$
\chi(T, x)=v^{\tau}(x), \quad \chi\left(T^{-1}, x\right)=v^{* \tau}(x) .
$$

Nun setzen wir

$$
R=R_{1}\left(\begin{array}{ll}
V^{\prime-1} & 0 \\
0 & V
\end{array}\right)
$$

hierbei bezeichnet $\gamma$ eine nicht-singuläre $(d \tau) \times(d \tau)$ Matrix mit Elementen aus $K$. Dann geht $T$ in $V^{-1} T V$ über. Wegen Satz 1 kann man $V$ so wählen, $\mathrm{da} \beta$

mit

$$
V^{-1} T V=\left(\begin{array}{cc}
T_{1} & T_{12} \\
0 & T_{2}
\end{array}\right)
$$

$$
\chi\left(T_{1}, x\right)=v^{\tau-1}(x), \quad \chi\left(T_{2}, x\right)=v(x)
$$

gilt. Nun sieht man sofort, daß das durch (76) gegebene $R$ die Bedingungen (12), (14), (15) exfülltt.

6. Wir kommen zu (69). Indem man eventuell noch $M$ durch $-M$ ersetzt, erkennt man, daß es ausreicht, den Fall

$$
\chi(M, x)=(x-1)^{2 n t}
$$

zu betrachten. Offenbar gilt $h(x)=h^{*}(x)=(x-1)^{\alpha}$ mit einer natürlichen Zahl $a$. Es genügt wieder, den Fall $\alpha=1$. zu untersuchen, weil sich daraus der allgemeine Fall sofort durch Indulition nach $a$ ergibt.

$$
\text { Es ist also zu zeigen, daß es eine Matrix } R \in \Sigma(m, K) \text { mit }
$$

$$
R^{-1} M R \in \Sigma_{1}(m, K)
$$

gibt. Zerlegt man dann $R^{-1} M R$ entisprechend (12) in Kästchen, so erfüllen diese von selbst die Gleichungen (14), (15).

Es sei $J(\mu)$ die $\mu \times \mu$ Matrix der Gestalt

$$
J(\mu)=\left(\begin{array}{cccc}
1 & 1 & & \\
\ddots & \ddots & 0 \\
0 & \ddots & \cdot & 1 \\
& & & \cdot 1
\end{array}\right)
$$

Wegen [2, Lemma 5], brauchen wir nur die beiden folgonden Möglichkeiton zu. untersuchen: Es gilt

$$
Q^{-1} M Q=J(2 m)
$$

mit einer nicht-singulären $(2 m) \times(2 m)$ Matrix $Q$, deren Elomente in $K$ liegen. Oder es ist

$$
Q^{-1} M Q=\left(\begin{array}{ll}
J(m) & 0 \\
0 & J(m)
\end{array}\right)
$$

für

$$
m \equiv 1 \bmod 2 \text {. }
$$

Dabei bezeichnet $Q$ wieder eine nicht-singuläre $(2 m) \times(2 m)$ Matrix mit Elementen aus $K$.

Im Falle (82) können wir wegen [2, (182)],

$$
M=\left(\begin{array}{ll}
J(m) & * \\
0 & J^{\prime-1}(m)
\end{array}\right)
$$

annehmon. Nun folgt (80) mit

$$
R=\left(\begin{array}{ll}
U & 0 \\
0 & U^{\prime-1}
\end{array}\right)
$$

hierbei ist $U$ eine nicht-singuäre Matrix, für die

$$
U^{-1} J(m) U=J^{\prime}(m)
$$

gilt. Die Elemente von $U$ lassen sich rational wählen.

Im Falle (83) können wir wegen [2, Lemma 6],

$$
M=\left(\begin{array}{ll}
J^{\prime}(m) & 0 \\
0 & J^{-1}(m)
\end{array}\right)
$$

annehmen. Jetzt folgt (80) mit $R=E$.

7. Wir krommen zum Fall (70).

HInFssatiz 1 . Ds sei $N$ eine $k \times l$ Matrix mit Elementen aus $K$, die den Bedingungen

$$
\begin{gathered}
N^{\prime} I(k) N=0, \\
\operatorname{Rang} N=l, \\
2 l \leqslant \hbar
\end{gathered}
$$

genügt. Dann zann man $N$ zin einer Natrix aus $\Sigma(k, K)$ ergänzen.

Boweis. Wir fassen uns kuxz, da der Beweis nach der in [3] angegebenen. Methode verläuft. Offenbar bleiben die Bedingungen (89), (90) richtig, wenn man $N$ von link mit einer Matrix auss $\Sigma(k, K)$ multipliziert. Durch Multiplikation mit einer Matrix aus $\Sigma(k, K)$ erreicht man, daß die erste Spalte von $N$ die erste Binheitsspalte wird. Wie in [3], (20), folgt dann

$$
N=\left(\begin{array}{ll}
1 & b_{1} \\
0 & B_{1} \\
0 & 0 \\
0 & O_{1}
\end{array}\right)
$$


wobei die Matrix $\left(\begin{array}{l}B_{1} \\ C_{1}\end{array}\right)$ den Bedingungen (89), (90) mit $k-1, l-1$ statt $k, l$ genügt. Nunmehr folgt Hilfssatz 1 induktiv nach der in [3] angegebenen Methode.

Man ergänze die in (25), (26), (27) auftretende Matrix $N$ zu einer Matrix $\tilde{R}_{1}=\left(N N_{1}\right) \in \Sigma(c s, K)$. Dann gilt $\tilde{R}_{1}^{-1} N=\left(\begin{array}{l}\mathbb{Q} \\ 0\end{array}\right)$. Hieraus und aus (25) folgt dann (53). Anwendung von (52) liefort (51). Da die Matrix (51) symplektisch ist, folgt

$$
\left(\begin{array}{lllr}
\tilde{P}^{\prime} & * & * & * \\
0 & * & * & * \\
0 & 0 & \tilde{P}^{-1} & * \\
0 & * & * & *
\end{array}\right) .
$$

Indem wir (93) mit dem. Inversen von (50) konjugieren, bekommt man (49). Damit ist die Lösbarkseit von (12) bewiesen. Die Bedingungen (1.4), (15) folgen aus (24).

8. Hrrifssanz 2. Es sei $K \in \Sigma(n, Q)$. Dann gibt es eine Modulmatrix $R \in \Gamma(n)$ mit

$$
R^{-1} K \epsilon \bigcap_{v=1, \ldots, n} \Sigma_{y}(n, Q)
$$

Beweis. Dieser Hilfssatz folgt aus einem Resultat von M. Koecher [5] man kann ihn aber auch wie folgt leicht beweisen. Für $n=1$ ist die Behauptung richtig, da es ein $R \in \Gamma(1)$ mit

$$
R^{-1} K=\left(\begin{array}{ll}
* & * \\
0 & *
\end{array}\right)
$$

gibt. Nun sei $n>1$ und die Behauptung für $n-1$ statt $n$ bewiesen. Wie man aus H. Klingen [4, Hilfssatz 2] oder aus [1, Hilfasatz 6] entnimmt, gibt es ein $R_{1} \in \Gamma(n)$ mit

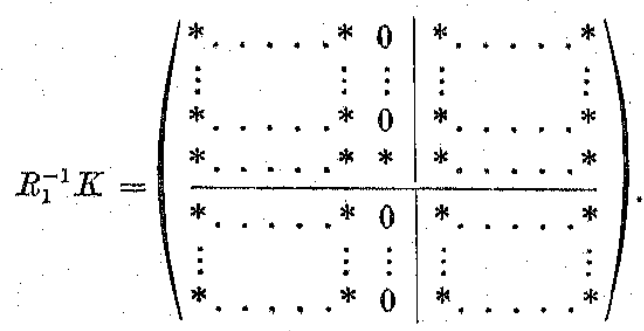

Da diese Matrix symplektisch ist, folgt

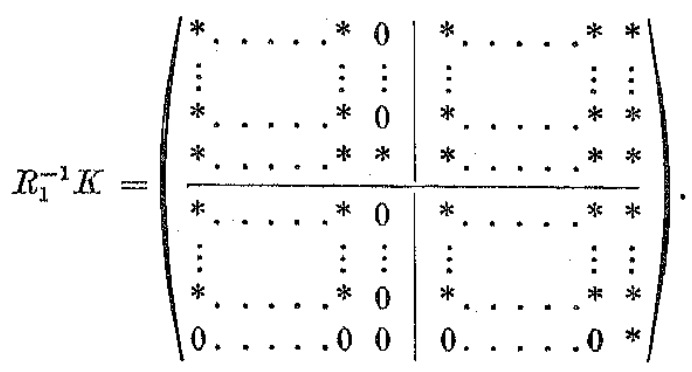

Nunmehr orgibt sich Hilfssatz 2 durch Induktion. Jetzt sei $M \in \Sigma(n, Q)$ and $K \in \Sigma(n, Q)$, so daß

$$
K^{-1} M K \in \Sigma_{j}(n, Q)
$$

gilt. Aas (94) folgt $K=R L$ mit $R \in \Gamma(n)$ und

$$
L \in \bigcap_{v=1, \ldots, n} \Sigma_{v}(n, Q) \subset \Sigma_{j}(n, Q) \text {. }
$$

Also

(98)

$$
R^{-1} M R \in \Sigma_{j}(n, Q)
$$

Satz 2 ist bewiesen.

9. Wir wollen jetzt die Lösbarkeit der Bedingungen (25), (26), (27) durch eine $(2 c s) \times(2 c t)$ Matrix $N$ mit Elementen aus $K$ untersuchen. Dazu seien

$$
p(x)=x^{2 c}+a_{2 c-1} x^{2 c-1}+\ldots+a_{1} x+1
$$

ein irreduzibles involutorisches Polynom aus $K[x]$ mit

$$
\text { (100) } \quad a_{\nu}=a_{2 c \rightarrow p} . \quad(\nu=1, \ldots, 2 c-1)
$$

und.

$$
\alpha_{1}, \ldots, \alpha_{2 c}
$$

dio Nullstellen ron $p(x)$. Bei pasizender Indizierung der Nullstellen gilt

$$
a_{2 a-r+1}=a_{v}^{-1} \quad(v=1, \ldots, c) \text {. }
$$

Man bilde die $(20) \times(20)$ Matrizen 
und

$$
\hat{A}=\left(\begin{array}{cccccc}
\alpha_{1} & & & & & \\
& \ddots & & & & 0 \\
& & \alpha_{c} & & & \\
& & & \alpha_{c}^{-1} & & \\
& 0 & & & \cdot & \\
& & & & & \alpha_{1}^{-1}
\end{array}\right)
$$

Dann gibti es cine nicht-singuläre $(20) \times(20)$ Matrix mit Elementen aus $K\left(a_{1}, \ldots, a_{2 c}\right)$, so $\mathrm{da} \beta$

$$
\text { (105) } \quad G^{-1} A Q=\hat{A}
$$

gilt. so $d a \beta$

Hicmssatz 3 . Es sei $X$ eine $(20) \times_{*}(2 c)$ Matrix mit Dlementen aus $K$,

$$
A X=\not A
$$

gitt. Die Gesamtheit der Lösungen $X$ von (106) bildet einen (2c)-dimensionaten Vektorraum über $K$ mit der Basis

$$
D, A, A^{2}, \ldots, A^{20-1} \text {. }
$$

Es gilt entweder

$$
X=0 \text { oder } \operatorname{Det} X \neq 0 \text {. }
$$

Beweis. Man' setze $\hat{X}=G^{-1} X G$. Die Formel (106) stellt ein homogenes lineares Gleichungssystem für $X$ dar. Um die Dimension des Lösungsraumes zu berechnen, genügt es also, die Dimension des Lösungsraumes von.

$$
\hat{A} \hat{X}=\hat{X} \hat{A}
$$

zu bestimmen. Offenbar ist (109) genau dann exfüllt, wenn $\hat{X}$ eine Dian gonalmatrix ist. Die Dimension des Lösungsraumes von (109) ist also $2 e$.

Es seien $l_{0}, l_{1}, \ldots, l_{20-1} \in K$ and $l_{0}, \ldots, l_{2 c-1}$ nicht alle Null. Wir raigen, daß dann die Determinanto von

$$
l_{0} E+l_{1} A+\ldots+l_{2 c-1} A^{2 c-1}
$$

nicht verschwindet. Zum Beweis konjugicren wix (110) mit $Q$ und bekommen die Diagonalmatrix

$$
l_{0} E+l_{1} \hat{A}+\ldots+l_{2 c-1} \hat{A}^{2 c-1} .
$$

Das $v$-te Diagonalelement von (111) ist

$$
l_{0}+l_{1} \alpha_{\nu}+\ldots+l_{2 c-1} a^{2 c-1} .
$$

Dieses laann nicht vorschwinden, da sonst $\alpha_{\nu}$ Nullstelle eines Polynoms aus $K[x]$ vom Grade $\leqslant 20-1$ wäre. Das widerspricht der Tatsache, daß $\alpha$, die Nullstelle des irreduziblen Polynoms $p(x)$ vom Grade $2 c$ ist. Somit folgt, daß die Determinante von (11.0) nicht Null ist. Insbesondere sind die Matrizen (107) über $K$ linear unabhängig; ferner gilt (108). Hilfssatz 3 ist bewiesen.

10. Ws sei $\mu$ eine natiüliche Zahil. Wir bilden die $(2 c \mu) \times(2 c \mu)$ Matrizen

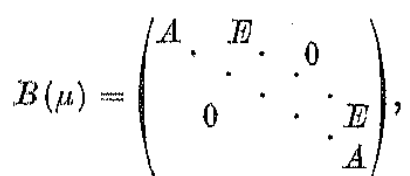

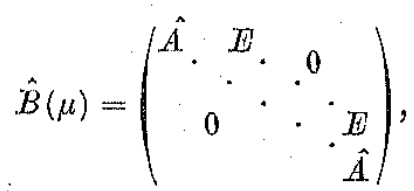

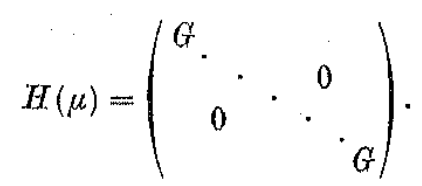

Dann gilt

$$
H^{-1}(\mu) B(\mu) H(\mu)=\hat{B}(\mu)
$$

sowie

$$
\text { (117) } \quad \operatorname{Rang}\left(\alpha_{\nu}, E-B(\mu)\right)=2 c \mu-1 \quad(\nu=1, \ldots, 2 c) \text {; }
$$

hierbei sind die $\alpha_{p}$ durch (101) gegeben.

11. Nun sei $M \in \Sigma(c s, K)$ und $P$ eine nicht-singuläre $(2 c t) \times(2 c t)$ Matrix mit Elementen aus $K$, so daß

$$
\begin{aligned}
\chi(M, x) & =p^{s}(x), \\
\chi(P, x) & =p^{t}(x)
\end{aligned}
$$

gilt. Gesucht ist cine $(20 s) \times(2 c t)$ Matrix $N$ mit

$$
M N=N P \text {, }
$$

so daß (26), (27) erfült sind. Man konjugiere Mr mit einer Matrix aus $\Sigma(e s, K)$ und $P$ mit einer nicht-singuläjen $(2 c t) \times(2 c t)$ Matrix, deren Elemento in $K$ liegen. Damn wird $N$ von links mit einer Matrix aus $\Sigma(o s, K)$ und von rechts mit einer wicht-singulären $(2 c t) \times(2 c t)$ Matrix multipliziert, deren Elemente aus $K$ sind. (26), (27) bleiben dabei ungeändert. Auf Grund der in [2] entwickelten Reduktionstheorie kann man also

$$
M=\left\{M_{1}, \ldots, M_{r}\right\} \in \Sigma\left(c s_{1}, \ldots, c s_{r} ; K\right)
$$


mit

$$
\chi\left(M_{0}, x\right)=p^{\varepsilon_{e}(x)} \quad(\varrho=1, \ldots, r)
$$

und

(123)

$$
s_{1}+\ldots+s_{r}=s
$$

erreichen. Wegen $[2,(105)]$, gibt es dabei zu jedem $M_{e}$ eine aicht-singuläre $\left(2 c s_{Q}\right) \times\left(2 c s_{e}\right)$ Matrix $Q_{e}$ mit Elementen aus $K$, so daß

$$
Q_{e}^{-1} M_{e} Q_{Q}=B\left(s_{Q}\right) \quad(\varrho=1, \ldots, r)
$$

gilt. Weiter kann man

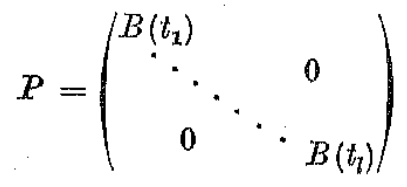

mit

$$
t_{1}+\ldots+t_{l}=t
$$

annehmen. Man setze

$$
F=\left(\begin{array}{ccccccc}
E_{1} & 0 & 0 & 0 & \ldots & 0 & 0 \\
0 & 0 & \mathbb{E}_{2} & 0 & \ldots & 0 & 0 \\
\ldots & \ldots & \ldots & \ldots & \ldots & \ldots & 0 \\
0 & 0 & 0 & 0 & \ldots & \mathbb{E}_{r} & 0 \\
0 & \mathbb{E}_{1} & 0 & 0 & \ldots & 0 & 0 \\
0 & 0 & 0 & \mathbb{E}_{2} & \ldots & 0 & 0 \\
\cdots & \ldots & \ldots & \ldots & \ldots & \ldots & \ldots \\
0 & 0 & 0 & 0 & \ldots & 0 & E_{r}
\end{array}\right)
$$

Hierbei ist $\mathbb{E}_{\mathrm{a}}$ die $\left(c s_{\mathrm{Q}}\right) \times\left(c s_{\mathrm{Q}}\right)$ Einheitsmatrix $(\varrho=1, \ldots, r)$. Es gilt

$$
F^{\prime}=F^{-1}
$$

wegen (121) ferner

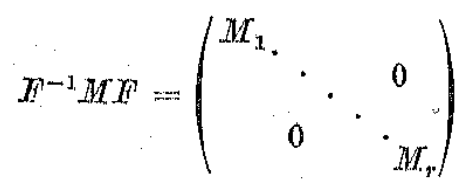

und schlieBlich

(130)

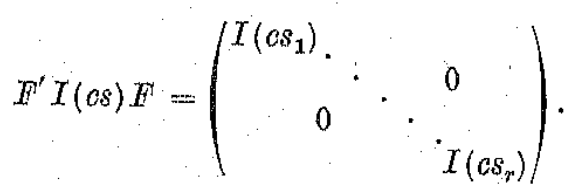

Man bilde die Matrizen

Aus (26), (27) folgt

(131)

Es gilt

und

$$
S_{Q}=Q_{Q}^{\prime} I\left(c s_{Q}\right) Q_{e} \quad(\varrho=1, \ldots, r) .
$$

letzteres folgt aus $M M_{e}^{\prime} I\left(c s_{e}\right) M_{Q}=I\left(c s_{e}\right)$ und (124). Wir setzen

$$
L=\left(\begin{array}{lll}
Q_{1} & & \\
& \ddots & 0 \\
0 & \ddots & Q_{r}
\end{array}\right)^{-1} F^{-1} N .
$$

Die Gleichung (120) geht über in

$$
\left(\begin{array}{ccc}
B\left(s_{1}\right) & & \\
0 & \ddots & 0 \\
& & { }_{B\left(s_{r}\right)}
\end{array}\right) L=L P .
$$

$$
L^{\prime}\left(\begin{array}{ccc}
S_{1} & & \\
& \ddots & 0 \\
0 & & S_{r}
\end{array}\right) I=0
$$$$
\text { Rang } L=2 c t \text {. }
$$

Genïgt andererseits $L$ den Bedingungen (136), (137), (138), so definiere man $N$ durch (135). Dieses $N$ erfïllt (25), (26), (27).

Wir zeigen jetzt, daß die Matrizen $S_{q}$ ähnliche Gestalt besitzen, wie die Matrizen $[2,(115)]$ und $[2,(153)]$.

Hrrimssatz 4. Jede Matrico $S_{\mathrm{e}}$ besitzt die Gestalt 
Hierbei sind die $S_{a t x}\left(\iota, x=1, \ldots, s_{\ell}\right)$ Matrizen von 20 Zeilen und Spatten. Es gilt

$$
\begin{aligned}
& \text { (140) } \quad A^{\prime} S_{e t x} A=S_{e t x} \quad\left(\iota, x=1, \ldots, s_{e}\right) \text {, } \\
& \text { (14I) } \quad S_{e x x}=-S_{u x t}^{\prime} \quad\left(\iota, x=1, \ldots, s_{e}\right) \text {, } \\
& \text { (142) } \quad \operatorname{Det}_{e, t, s_{0}-\imath+1} \neq 0 \quad\left(\iota=1, \ldots, s_{\ell}\right) \text {, } \\
& \text { (143) } \quad S_{Q, t+1, s_{e}-t}=-A^{\prime-1} S_{Q, t, s_{Q^{-}-t+1}} A \quad\left(\iota=1, \ldots, s_{e}-1\right) \text {. }
\end{aligned}
$$

Beweis. Wir lassen für die Rechnung den Index $\varrho$ fort. Insbessondere schreiben wir $s$ statt $s_{\dot{e}}$. Man setze.

$$
\begin{gathered}
\hat{S}=H^{\prime}(s) S H(s) \\
S=\left(\begin{array}{ccc}
S_{11} & \ldots & S_{1 s} \\
\ldots & \ldots & \ddots \\
\hat{S}_{s 1} & \ldots & \hat{S}_{s s}
\end{array}\right), \quad \hat{S}=\left(\begin{array}{lll}
\hat{S}_{11} & \ldots & \hat{S}_{1 s} \\
\ldots & \ldots & \hat{S}_{s s} \\
\hat{S}_{s 1} & \ldots & \hat{S}_{s s}
\end{array}\right), \\
\hat{S}_{t x}=G^{\prime} S_{b x} G \quad(\iota, x=1, \ldots, s) .
\end{gathered}
$$

Aus (134) folgt dann

$$
\hat{B}^{\prime}(s) \hat{S} \hat{B}(s)=\hat{S} .
$$

Wegen (114) ist (147) gleichbedeutend mit

$$
\begin{gathered}
\hat{A}^{\prime} \hat{S_{11}} \hat{A}=\hat{S}_{11} \\
\hat{A}^{\prime} \hat{S}_{1, \kappa-1}+\hat{A}^{\prime} \hat{S_{1 x}} \hat{A}=\hat{S_{1 *}} \quad(x=2, \ldots, s),
\end{gathered}
$$$$
\text { (1.50) } \quad \hat{S}_{t-1,1} \hat{A}+\hat{A}^{\prime} \hat{S}_{t 1} \hat{A}=\hat{S}_{\imath 1} \quad(\iota=2, \ldots, s),
$$$$
\text { (151) } \quad \hat{S}_{\iota-1, x-1}+\hat{A}^{\prime} \hat{S}_{t, x-1}+\hat{S}_{l-1, x} \hat{A}+\hat{A}^{\prime} \hat{S}_{\imath x} \cdot \hat{A}=\hat{S}_{\iota x} \quad(\iota, x=2, \ldots, s) \text {. }
$$

Aus (104), (148) ersieht man, daß $\hat{S}_{11}$ die Gestalt

$$
\left(\begin{array}{ccc}
0 & . \cdot & * \\
* & \cdot & 0
\end{array}\right)
$$

besitzt. Wegen (149) folgt dann durch Indudtion nach $x$, daß $\hat{S}_{1 x}(x=2, \ldots$ $\ldots, s)$ ebenfalls von der Form (152) ist. Dasselbe belkommt man für $\hat{S}_{\iota 1}^{\prime}$ $(\iota=2, \ldots, s)$ ans $(150)$ durch. Induktion nach $\iota$. Sehließlich crgibt sich dann aus (151), daß alle $\hat{\hat{S}}_{t *}$ von der Gestalt (152) sind, indem man erst Induktion nach dem ejnen und dann nach dom anderen Index macht. Also ist jedes $\hat{S}_{\iota k}(\iota, x=1, \ldots, s)$ von der Form (152). Daraus folgt

$$
\hat{A}^{\prime} \hat{S}_{c k} \hat{A}=\hat{S}_{i x} \quad(\iota, x=1, \ldots, s) \text {. }
$$

Anwendung von (105), (146) liefert (140). Aus (149), (150), (151) einerseits und. (153) andererseits bekommt man. weiter

$$
\begin{gathered}
\hat{S}_{1, x-1}=0, \quad \hat{S}_{\iota-1,1}=0 \quad(\iota, x=2, \ldots, s), \\
\hat{S}_{\iota-1, x-1}+\hat{A}^{\prime} \hat{S}_{\iota, x-1}+\hat{S}_{\iota-1, x} \hat{A}=0 \quad(\iota, x=2, \ldots, s) .
\end{gathered}
$$

Hicrans schließt man.

$$
\hat{\mathcal{S}}_{i x}=0 \quad(\iota+x \leqslant s) .
$$

Wegen (146) folgen hieraus (139) und (143). Die Formeln (141) und (142) bekommt man aus (132), (133), (1.39). Hilfssatz 4 ist bewiesen.

12. Wir zerlegen die in (136) anftretende Matrix $x$ in der Gestalt (157)

$$
L=\left(L_{Q \lambda}\right) \quad(\varrho=1, \ldots, r ; \lambda=1, \ldots, l)
$$

mit $\left(2 e s_{Q}\right) \times\left(2 c t_{\lambda}\right)$ Kästchen $L_{e \lambda}$. Dann ist (136) gleichbedeutend mit

(158) $\quad B\left(s_{e}\right) \dot{L}_{0 \lambda}=L_{0 \lambda} B\left(t_{\lambda}\right) \quad(\varrho=1, \ldots, r ; \lambda=1, \ldots, l)$.

Der folgende Hilfssatz erinnert an [2, (94) $)]$.

HThFssatz 5. Die Matrio $L_{Q \lambda}$ ist genau dann eine Lösung von (158), wenn gilt

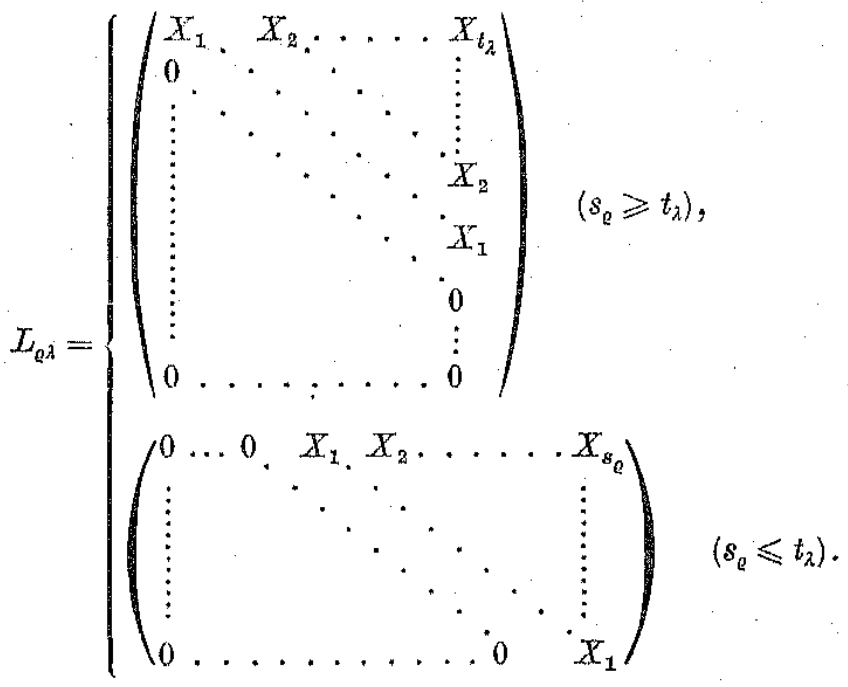

Hierbei sind $X_{1}, X_{2}, \ldots$ mit A vertauschbare $(2 c) \times(2 c)$ Matrizen, deren Dlemente in K Tiegen.

Beweis. Man schreibe $L$ statt $L_{e \lambda}$ und $s, t$ statt $s_{Q}$ und $t_{\lambda}$. Ferner sei

$$
L=\left(\begin{array}{ccc}
X_{11} & \ldots & X_{1 t} \\
\ldots & \ldots & \ddots \\
X_{s 1} & \ldots & X_{s t}
\end{array}\right)
$$


mit $(2 c) \times(2 e)$ Matrizen $X_{\sigma \tau}\left(\sigma^{\prime}=1, \ldots, s ; \tau=1, \ldots, t\right)$. Weiter werde

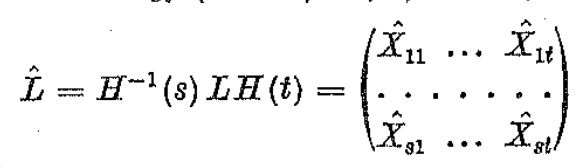

\section{gesetzt. Dann gilt}

$$
\text { (162) } \quad \hat{X}_{\sigma r}=G^{-1} X_{\sigma x} G \quad(\sigma=1, \ldots, s ; \tau=1, \ldots, t) \text {. }
$$

Aus (158) ergibt sich

$$
\hat{B}(s) \hat{L}=\hat{L} \hat{B}(t)
$$

Das ist äquivalent zu folgenden Gleichungen:

(164) $\hat{A} \hat{X}_{\sigma \tau}+\hat{X}_{\alpha+1, \tau}=\hat{X}_{\sigma, \tau-1}+\hat{X}_{\sigma \tau} \hat{A} \quad(\sigma=1, \ldots, s-1 ; \tau=2, \ldots, t)$,

$$
\begin{gathered}
\hat{A} \hat{X}_{\sigma 1}+\hat{X}_{\sigma+1,1}=\hat{X}_{\sigma 1} \hat{A} \quad(\sigma=1, \ldots, s-1), \\
\hat{A} \hat{X}_{s \tau}=\hat{X}_{s, \tau-1}+\hat{X}_{a t} \hat{A} \quad(\tau=2, \ldots, t), \\
\hat{A} \hat{X}_{s 1}=\hat{X}_{s 1} \hat{A} .
\end{gathered}
$$

Aus (104) und (167) ersieht man, daß $\hat{X}_{81}$ eine Diagonalmatrix ist. Wegen $(165),(166)$ sind dann auch $\hat{X}_{\sigma 1}$ und $\hat{X}_{s x}$ Diagonalmatrizen. Schließlich liefert (164), daß alle $\hat{X}_{\sigma \tau}$ Diagonalmatrizen sind. Also

(168)

$$
\hat{A} \hat{X}_{\sigma \tau}=\hat{X}_{\sigma \tau} \hat{A} \quad(\sigma=1, \ldots, s ; \tau=1, \ldots, t) .
$$

Auf Grund ron (105), (162) folgt

$$
A X_{\sigma \tau}=X_{\sigma \tau} A \quad(\sigma=1, \ldots, s ; \tau=1, \ldots, t) .
$$

Anwendung von (168) auf (164) bis (166) liefert wegen (162):

$$
\begin{gathered}
X_{\sigma+1, \tau}=X_{\sigma, \tau-1} \quad(\sigma=1, \ldots, s-1 ; \tau=2, \ldots, t), \\
X_{21}=\ldots=X_{s 1}=0 \\
X_{s 1}=\ldots=X_{s, t-1}=0 .
\end{gathered}
$$

Daraus folgt (159). Nun sieht man leicht, daß die durch (159) gegebenen $I_{Q \lambda}$ auch (158) lösen, wenn $X_{1}, X_{2}, \ldots$ mit $A$ vertauschbar sind. Filfssatz 5 ist bewiesen.

Durch Hilfssatz 5 haben wir einen vollstiändigen Utberblick über alle Lösungen von (136). Wegen (157) ist (137) äquivalent zu.

$$
\sum_{\varrho=1}^{r} L_{\varrho x}^{\prime} S_{\varrho} L_{\varrho \lambda}=0 \quad(x, \lambda=1, \ldots, l) .
$$

Wir werden nun sehen, daß (173) zusammen mit (138) in einigen Fällen lösbar in anderen Fällen unlösbar ist.
13. SATZ 3. Es sei

und

$$
\begin{gathered}
r \geqslant l \\
s_{\lambda} \geqslant 2 t_{\lambda} \quad(\lambda=1, \ldots, \dot{l}) .
\end{gathered}
$$

Dann sind die Bedingungen (136), (137), (138) Zösbar. Beweis. Aus (139), (159) folgt

Wir wählen nun

$$
I_{e x}^{\prime} S_{\varrho} L_{e^{\lambda}}=0 \quad\left(s_{\varrho} \geqslant t_{x}+t_{\lambda}\right)
$$

$$
\begin{gathered}
L_{\varrho \lambda}=0 \quad(\varrho \neq \lambda), \\
I_{\lambda \lambda}=\left(\begin{array}{c}
E \\
0
\end{array}\right) \quad(\lambda=1, \ldots, l)
\end{gathered}
$$

mit einer $\left(2 c t_{\lambda}\right) \times\left(2 c t_{\lambda}\right)$ Einheitsmatrix $E$. Dann gilt

$$
x_{Q x}^{\prime} S_{\varrho} x_{Q \lambda}=0 \text {, }
$$

falls nicht $x=\varrho=\lambda$ gilt. Bei $x=\varrho=\lambda$ ergibt sich aber (179) aus (175) , und (176). Also ist (173) exfüllt. Die Matrizen (177), (178) besitzen fernèr die Gestalt (159); sie lösen deshalb (158). Man füge die Kästchen $L_{0 \lambda}$ entsprechend (1.57) zu einer Matrix $L$ zusammen; diese exfüllen dann: die Bedingungen (136), (137). Aus der Konstruktion von $L$ folgt weiter die Gültigkeit von (138). Satz 3 ist bewiesen.

14. Wir zeigen jetzt an zwei Beispielen, daß (136), (137), (138) nicht immer lösbar sind.

Berspren 1. Es sei

$$
\begin{gathered}
r=2, l=1 \\
s_{1}=s_{2}=t_{1}=1
\end{gathered}
$$

Aus (157), (159) folgt dann

$$
L=\left(\begin{array}{l}
X_{1} \\
X_{2}
\end{array}\right)
$$

wobei $X_{1}$ und $X_{2}$ mit $A$ vertauschbar sind. Jedes solche $L$ löst (136). Aus (173) belkommt man

$$
X_{1}^{\prime} S_{1} X_{1}+X_{2}^{\prime} S_{2} X_{2}=0 .
$$

Hierbei sind $S_{1}, S_{2}$ schiefsymmetrisch und nicht singulär.

Wir behaupten weiter, daß die Bedingung (138) zu

$$
\operatorname{Det} X_{1} \neq 0, \quad \operatorname{Det} X_{2} \neq 0
$$

äquivalent ist. Aus (184) folgt jedenfalls (138). Wir nehmen jetzt an, daß (184) nicht gilt und zeigen die Ungültigkeit von (138). Ohne Beschränkung der Allgemeinheit sei Det $X_{1}=0$, wegen Hilfssatz 3 also $X_{1}=0$. 
Die Gleichung (183) liefert dann $X_{2}^{\prime} S_{2} X_{2}=0$; aber Det $S_{2} \neq 0$, somit Det $X_{2}=0$, d.h., $X_{2}=0$ auf Grund von Hillessatz 3. Bei $X_{1}=X_{2}=0$ gilt aber (138) nicht. Damit ist die Behauptung bewiesen.

Es bleibt also festzustellen, wann die Bedingung (183) gilt. Man setze $X=X_{2} X_{1}^{-1}$. Dann ist $X$ mit $A$ vertauschbar und aus (183) folgt (185)

$$
S_{1}+X^{\prime} S_{2} X=0 \text {. }
$$

Ferner hat man

$$
\operatorname{Det} X \neq 0
$$

Es gebe andererseits oin mit $A$ vertathchbares $X$, so dak die Beziehungen (185), (186) gelten. Dann ist $X_{1}=E, X_{2}=X$ eine Iüsung von (183). Man braucht also nur noch festzustellen, wann (185) durch ein nichtim singuläres mit $A$ vertauschbares $X$ lösbar ist. Dieses Problem führt nun genau auf die in [2, Seiten 230-232] beschriebene Reduktionstheorie. Daraus entnimmt man, daß (185) genau dann lösbar ist, wenn gewisse arithmetische Bedingungen gelton. Im Falle (180), (181) führt also die Frage nach der Lösbarkeit von (136), (137), (138) auf arithmetische Bedingungen.

Brispiac 2. In diesex Arbeit tritt das Problem der Lösbarkeit von (136) bis (138) nur für $s \geqslant 2 t$ auf. Zerlegt man aber $L$ in Kästchen der Gestalt (157), so kann dabei auch $s_{e}<2 t_{\lambda}$ sein. Deshalb betracliten wir auch den Fall

(187)

$$
r=l=1
$$

mit

$$
s<2 t
$$

und zeigen, daß es dann keine Lösung $L$ von (136), (137), (138) gibt.

Wegen (138), (159) existiert jedenfalls keine Lösung bei $s<t$. Für $t \leqslant s<2 t$ hat man

$$
L=\left(\begin{array}{ccc}
x_{1} & \cdots & X_{t} \\
0 & \ddots & \vdots \\
\vdots & \ddots & X_{1} \\
\vdots & & 0 \\
\vdots & & \vdots \\
0 & \cdots & 0
\end{array}\right)
$$

wobei $X_{1}, \ldots, X_{t}$ mit $A$ rentauschbar sind. (138) ist dann gleichbedeutend mit

(190)

$$
\operatorname{Det} X_{1} \neq 0 \text {. }
$$

Wegen $r=1$ gibt es nur eine Matrix $S$ der in (13.1) genannten Art. Formel (137) geht über in

$$
L^{\prime} S L=0
$$

Wegen (139) gilt dabei

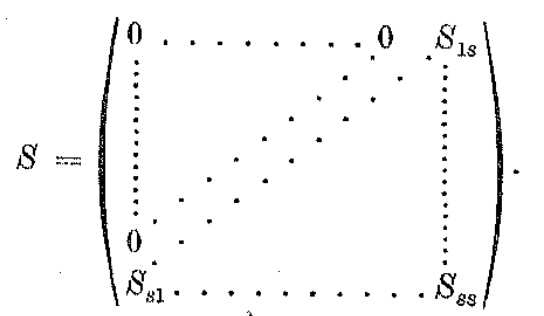

Berechnet man nun $L^{\prime} S L$ aus (189) und (192), so sieht man, daß eines der $(2 c) \times(2 c)$ Kästchon von $X^{\prime} S L$ die Gestalt $X_{1}^{\prime} S_{s-t+1, t} X_{1}$ besitzt. Aus (142), (190) folgt $\operatorname{Det}\left(X_{1}^{\prime} S_{s-t_{+1}, t} X_{1}\right) \neq 0$. Somit kann (191) nicht gelten. D.h., (136) bis (138) sind unlösbar.

\section{Literatur}

[1] U. Christian, Einfïhrung in die Theorie der paramodularen Gruppen, Math. Ann. 168 (1967), S. 59-104.

[2] - A reduction theory for sympleotic matrices, Math. Zeitschr. I01 (1967), S. 213244.

[3] - Uber die erste Zoile paramodularer Matrizen, Nachr. Akad. Wiss. Göttingen, math.-phys: Klasse, 1967, \$. 239-245.

[4] H. Klingen, Über die Eirseugenden gewisser Modulgruppenn, Nachr. Akad. Wiss. Gö̈tingen, math.-phys. Klasso, 1956, S. 173-185.

[5] M. Koecher, Zur Theorie der Modulformen n-ten Grades II, Math. Zeitschr. 61 (1955), S. $455-466$.

[6] G. Z. Wall, On the conjugacy classes in the unitary, symplectic and orthogonal groups, J. Australian Math. Soc. 3 (1963), S. I-62. 